\title{
Extremely Large Telescopes on the Antarctic plateau
}

\author{
J.S. Lawrence
}

School of Physics, University of New South Wales, NSW 2052, Australia

\begin{abstract}
The primary limitation to the performance of any large groundbased telescope is the atmospheric properties of its site, particularly the sky emission and the turbulence structure. There are several sites on the Antarctic plateau (South Pole, Dome $\mathrm{C}$ and Dome A) for which the increase in infrared sensitivity relative to a mid-latitude site should be as much as two orders of magnitude. The unique turbulent structure above Dome $\mathrm{C}$ indicates that an extremely large telescope equipped with only a natural guide star adaptive optics system should achieve equivalent resolution to a mid-latitude extremely large telescope with a multi-conjugate multi-laser guide star system.
\end{abstract}

Experimental data from a number of instruments, eg (Phillips et al. 1999), has shown that the winter time thermal sky emission above the South Pole station is as much as two orders of magnitude lower than found anywhere else on Earth. Additionally, data from radiosonde balloons measuring temperature and relative humidity have shown that the South Pole average winter time precipitable water vapor column density is $0.25 \mathrm{~mm}$ (Chamberlain et al. 2001). This is significantly lower than found at good quality mid-latitude sites such as Mauna Kea (1.6 $\mathrm{mm}$ average).

These factors result in a South Pole telescope being substantially more sensitive in the near to far-infrared than any other ground based telescope of the same size. The increased atmospheric transparency also results in a number of new windows opening up in the sub-millimetre and far-infrared.

There are several other sites on the Antarctic plateau that offer potentially superior sensitivity to even South Pole. These include the French/ Italian Dome $\mathrm{C}$ station at an elevation of $3250 \mathrm{~m}$, and Dome A, the highest point on the plateau at an elevation of $4200 \mathrm{~m}$. Atmospheric models for these high plateau sites have been developed based on temperature, pressure, and water vapor profiles inferred from South Pole aerological records, and high plateau Automatic Weather Station data. These models (giving atmospheric emission and transmission, and incorporating a telescope emission component) show that the relative increase in telescope sensitivity in going from South Pole to Dome A is largest in the near infrared, where a factor of 5-10 is expected. The benefits of a reduced atmospheric emission in the mid-infrared are somewhat offset by the significant contribution from telescope emission at these wavelengths. In the far-infrared and sub-millimetre, the lower water vapor expected at the higher sites results in a dramatic increase in atmospheric transparency compared with South Pole.

While the benefits of the South Pole low infrared sky background are well recognized, and the unique atmospheric turbulence profile (the majority of turbulence is confined within the lowest $300 \mathrm{~m}$ ) is potentially beneficial for some applications, the ground level seeing is relatively poor (1.8 arcsec in the visible) 
compared with quality mid-latitude sites. However, the local topography of the high plateau sites (both Dome A and Dome C lie on high points of the plateau) indicates that the turbulence within the ground level inversion layer should be lower in magnitude than observed at the South Pole due to the absence of katabatic winds. The integrated ground level seeing during summer at Dome $\mathrm{C}$ was shown to be a median of $1.2 \operatorname{arcsec}$ (Aristidi et al. 2003). The boundary layer turbulence is observed to drop below the detection threshold for a sonic radar instrument operating throughout winter of 2003, representing a contribution to the total seeing of less than 0.2 arcsec (Travouillon et al, 2003).

These results are used to define upper and lower bounds to the refractive index structure function profile for Dome $\mathrm{C}$ assuming that the contribution from the free-atmosphere to the total turbulence is the same as found at the South Pole. These profiles give an isoplanatic angle of greater than 10 arcsec, which is significantly larger than found at other sites (typically 2 arcsec at Mauna Kea). Additionally, the lack of strong winds observed throughout the Dome $\mathrm{C}$ atmosphere result in an atmospheric coherence time than is much longer than found elsewhere. These two factors (isoplanatic angle and coherence time) represent a serious limitation to the performance of any adaptive optics system on an extremely large telescope, and drive the need for multi-conjugate systems with many deformable mirrors and laser guide stars at mid-latitude sites.

An error budget for a $30 \mathrm{~m}$ telescope at Dome C has been calculated and compared with that specified for the $30 \mathrm{~m}$ CELT situated at Mauna Kea. The Dome $\mathrm{C}$ telescope, equipped with a single natural guide star system should achieve a wavefront error of $180-250 \mathrm{~nm} \mathrm{rms}$. This is equivalent to a multiconjugate, multi-laser guide star system operating with a Mauna Kea atmosphere, and is a significant improvement over the single low order adaptive optics error of $500 \mathrm{~nm}$ rms. It should be noted that the turbulence profiles modeled here represent a conservative estimate of the isoplanatic angle at Dome C. It will be at least another two years until the atmospheric profile throughout winter is confirmed.

The improvements in sensitivity and resolution achievable by locating an extremely large telescope at Dome $\mathrm{C}$ rather than a mid-latitude site are so substantial that they could compensate for any logistical disadvantage. Additionally, factors such as the very low ground wind speed, and the non-existent seismic activity are very important for mechanical and structural considerations.

\section{References}

Aristidi, E., Agabi, E., Vernin, J., Azouit, M., Martin, F., Ziad, A., and Fossat, E. 2003, A\&A, 406, L19

Chamberlain, R. A. 2001, J. Geophys. Research, 106, 20101

Phillips, A., Burton, M.G. , Ashley, M.C.B., Storey, J.W.V., Llyod, J.P., Harper, D.A., and Bally, J. 1999, AJ, 527, 1009

Travouillon et al. A\&A, 400, 1163 